\title{
Genome-scale metabolic model predicts carbon flux partitioning towards starch biosynthesis in storage root of cassava
}

\author{
P. Chiewchankaset ${ }^{1}$, W. Siriwat ${ }^{2}$, S. Kalapanulak ${ }^{2,3}$, T. Saithong ${ }^{2,3 *}$ \\ ${ }^{1}$ Division of Biotechnology, School of Bioresources and Technology, King Mongkut's University \\ of Technology Thonburi (Bang Khun Thian), Bangkok, Thailand \\ ${ }^{2}$ Systems Biology and Bioinformatics Research Group, Pilot Plant Development and Training Institute, \\ King Mongkut's University of Technology Thonburi (Bang Khun Thian), Bangkok, Thailand \\ ${ }^{3}$ Bioinformatics and Systems Biology Program, School of Bioresources and Technology, King Mongkut's \\ University of Technology Thonburi (Bang Khun Thian), Bangkok, Thailand \\ *e-mail: treenut.sai@kmutt.ac.th
}

Key words: genome-scale metabolic model (GMM), carbon flux partitioning, cassava

Motivation and Aim: Cassava is one of the top rank stable crops feeding at least 800 billion people each year. The great amount of starch containing in underground roots have made cassava being a promising carbohydrate source for world population. In contrast to its fascination, how starch is highly produced and accumulated in cassava roots is still uncovered, and become a challenge research question.

Methods and Algorithms: To disclose the mystery of cassava metabolism, the genomescale metabolic model (GMM) was developed to disentangle the complex metabolic processes of carbon assimilation in cassava roots. The constraint-based model of carbon assimilation pathway was constructed based upon flux balance analysis technique.

Results: The model of carbon assimilation covered 393 metabolites related to 352 biochemical reactions, and 116 the transport reactions required for mobilizing the metabolites between cytoplasm, plastid, and mitochondria subcellular compartments. The model was used to relate the intracellular carbon flux partition to the observed yield of storage roots grown in the field under rainfed condition. Optimized to root growth rate, the model successfully simulated carbon flux towards cellular biomass production that reflected the metabolic phenotype underlying the growing biomass as well as starch accumulation in storage roots of cassava. The model was literature validated and was supported by model sensitivity analysis.

Conclusion: The GMM of cassava storage roots, herein, is the first and crucial step for inquiring more insightful questions of carbon metabolism that yields superior starch production than other starchy crops.

Acknowledgements: Supported by partially supported by King Mongkut's University of Technology Thonburi (KMUTT) under the Higher Education Research Promotion and National Research University Project of Thailand, Office of the Higher Education Commission, Ministry of Education, Thailand, and by NRCT and NSTDA (P-17-52192). 